\title{
Relevance of Surgery in Stage IV Gastric Carcinoma*
}

\author{
Keishiro Aoyagi", Kikuo Kouhuji, Motoshi Miyagi, Junya Kizaki, Taro Isobe, Kousuke Hashimoto, \\ Kazuo Shirouzu
}

Department of Surgery, Kurume University School of Medicine, Kurume, Japan.

Email: " keishiro@med.kurume-u.ac.jp

Received November $28^{\text {th }}, 2012$; revised December $30^{\text {th }}, 2012$; accepted January $10^{\text {th }}, 2013$

\begin{abstract}
New anticancer drugs are being increasingly used for advanced and recurrent gastric cancer in many institutions. Therefore, the relative importance of surgery may have changed, and there may also be controversy as to whether patients with Stage IV gastric cancer should or not undergo surgical resection. The relevance of surgery in this population was studied. The relevance of surgery was studied in 304 cases of Stage IV gastric cancer who were treated at Kurume University Hospital from 1995 to 2009. Multivariate analysis showed that distant organ metastasis was significantly correlated with surgery. In Stage IV cases, chemotherapy and the number of Stage IV factors were independent prognostic factors. In surgery cases, venous invasion, chemotherapy, and residual tumor were independent prognostic factors. R0 was significantly higher in the surgery with chemotherapy group than in the chemotherapy alone group, but there was no significant difference in R1 or R2 cases between the surgery with chemotherapy group and the chemotherapy alone group. In R2 cases, use of a new drug was an independent prognostic factor. The rate of R0 was significantly higher in the preoperative chemotherapy group than in the surgery alone group. In preoperative chemotherapy cases, the S-1/cisplatin (CDDP) group had a 50\% 2-year survival rate, and these cases underwent postoperative chemotherapy using the S-1 regimen. A multimodal treatment is considered most effective for Stage IV gastric cancer, where this includes preoperative chemotherapy, surgery, and postoperative chemotherapy using the new anti-cancer drugs.
\end{abstract}

Keywords: Gastric Cancer; Stage IV; Surgery; Chemotherapy; Multimodal Treatment

\section{Introduction}

Gastric cancer is the second leading cause of cancerspecific mortality worldwide [1]. In Japan, gastric cancer is still the second most frequent cause of cancer-related death, despite advances in diagnosis and treatment. For patients with unresectable or recurrent gastric cancer, outcomes are extremely poor, with a median survival time of 3 to 5 months if untreated. Previous investigators have suggested that palliative resection for gastric cancer may confer symptomatic and/or survival benefits. However, the survival benefit has been theoretically limited to certain groups of patients or characteristics, such as younger age, Asian race, and limited spread of disease [2-4]. Data from the Dutch Cancer Trial showed that patients under 70 years of age with only one positive site of metastatic disease benefited from resection $[5,6]$. Only N3 or T4N2 of Stage IV gastric cancer according to the $13^{\text {th }}$ Edition Japanese Classification of Gastric Carcinoma is excluded from the Stage IV factors of the $14^{\text {th }}$ Edition $[7,8]$. According to the $14^{\text {th }}$ Edition Japanese Classification, Stage IV gastric cancer is defined as far

\footnotetext{
*The authors declared that they have no competing interests.

\#Corresponding author.
}

advanced gastric cancer which has distant metastases such as peritoneal metastases, liver metastases, distant lymph node metastases, peritoneal cytology positive for carcinoma cells, and other distant organ metastases. In general, patients with such far advanced gastric cancer have usually not been considered good candidates for surgery. However, new anticancer drugs and molecular targeting therapies such as S-1, CPT-11, taxanes, and trastuzumab for Her-2 positive cases are being increasingly used for advanced and recurrent gastric cancers. Thus, the relative importance of surgery might have changed, and there is controversy as to whether patients with Stage IV gastric cancer should or not undergo surgical resection. Therefore, the relationship between surgery and survival was examined in this population.

\section{Materials and Methods}

\subsection{Patients}

Between 1995 and 2009, 2145 patients with gastric cancer underwent therapy at Kurume University Hospital. The 304 cases (206 men, 98 women) defined as Stage IV according to the $14^{\text {th }}$ Edition Japanese Classification of 
Gastric Carcinoma [8], excluding multiple gastric cancer and remnant stomach cancer, were studied retrospectively. The patients' mean age was 64.8 years (range, 27 91 years). Bypass operation, gastrostomy, enterostomy, colostomy, and simple laparotomy were excluded from the surgery group.

\subsection{Clinical Variables}

A total of about 27 clinicopathological variables were investigated including sex (male or female), age ( $\geq 60$ or $<60$ years), macroscopic type (type $0,(1,2),(3,4)$ or 5$)$, site (L, M, or $U)$, tumor size $(\geq 100$ or $<100 \mathrm{~mm})$, the number of sites $(1,2$, or $\geq 3$ ), liver metastasis ( $\mathrm{H} 0$ or $\mathrm{H} 1$ ), peritoneal metastasis ( $\mathrm{P} 0$ or $\mathrm{P} 1)$, lymph node metastasis (N0, N1,2, or N3), distant lymph node metastasis (M0 (LYM) or M1 (LYM)), distant organ metastasis excluding liver metastasis, peritoneal metastasis, distant lymph node metastasis (M0 or M1), peritoneal cytology (CY0 or CY1), depth of invasion ((T1, T2, T3), T4a, or T4b), histology (differentiated type or undifferentiated type), stromal pattern (medullary type (med), intermediate type (int), or scirrhous type (sci)), tumor infiltration pattern (INFa, INFb, or INFc), lymphatic invasion (ly0, ly1,2, or ly3), venous invasion (v0,1 or v2,3), proximal margin (PM0 or PM1), distal margin (DM0 or DM1), surgery (or + ), chemotherapy $(-$ or + ), the number of Stage IV factors $(1$, or $\geq 2)$, postoperative or preoperative chemotherapy, lymph node dissection (D0,1, D2, or $\geq \mathrm{D} 2$ ), residual tumor (R0, R1, or R2), and new anticancer drugs such as S-1, irinotecan, and taxanes $(-$ or +$)$.

\subsection{Relationship between Surgery and Clinical Variables}

Associations between surgery and clinical variables were compared by univariate analysis using the $\chi^{2}$ test. Significant factors were extracted for further multivariate logistic regression analysis.

\subsection{Survival}

The disease-specific survival for each clinical variable was calculated using the Kaplan-Meier method for all cases, surgery cases, and R2 cases. Univariate analysis of factors thought to affect disease-specific survival was carried out using the log-rank test. The Cox proportional hazards model was used in the multivariate analysis of the factors determined to be significant for disease-specific survival on univariate analysis. Moreover, prognosis was compared between the surgery with chemotherapy group and the chemotherapy alone group.

\subsection{Preoperative Chemotherapy Cases}

A total of 22 Stage IV gastric cancer patients (19 men, 3 women) who underwent preoperative chemotherapy were studied. The mean age was 62.2 years (range, 41 - 73 years). Four regimens were used as preoperative chemotherapy in 21 cases, including 5-fluorouracil (FU)/cisplatin (CDDP) in 9, S-1/CDDP in 8, S-1/taxol (TXL) in 2, and $\mathrm{S}-1$ in 2 , and one patient was given various anticancer drugs over a long period.

\subsection{Statistical Analysis}

The statistical analyses were performed using statistical analysis software (SPSS II; IBM Co., Armonk, NY, USA). For all analyses, significance was defined as $P<$ 0.05 .

\section{Results}

\subsection{Clinicopathological Features}

With respect to Stage IV factors, there were 107 cases with peritoneal metastases (44.4\%), 63 (26.1\%) distant lymph node metastases, 40 (16.6\%) liver metastases, 22 (9.1\%) peritoneal cytology-positive, and $9(3.7 \%)$ distant organ metastases among the patients with one Stage IV factor; $52(17.1 \%)$ patients had 2 Stage IV factors, and $11(3.6 \%)$ had 3 or 4 Stage IV factors. There were 45 metas- tasis sites affecting the peritoneum, 39 affecting distant lymph nodes, 32 in liver, 17 in distant organs, and 1 cy- tology-positive case in patients with multiple Stage IV factors. Of the 26 cases with distant organ metastases, $9(34.6 \%)$ involved bone, 5 (19.2\%) involved bone marrow, and 3 (11.5\%) involved lung; there was ovary, spleen, and skin involvement in $2(7.7 \%)$ each, bone, lung, brain, and kidney involvement in 1 (3.8\%) each, and 17 of 26 cases with distant organ metastases had other Stage IV factors.

Postoperative chemotherapy was given to 134 (44.1\%) patients, surgery alone was performed in 83 (27.3\%), chemotherapy alone was given in $36(11.8 \%)$, no surgery and no chemotherapy were given in $26(8.6 \%)$, preoperative chemotherapy was given in $22(7.2 \%)$, and treatment status was unknown in $3(1.0 \%)$. There were 239 surgery cases, with a resection rate of $78.6 \%$.

\subsection{Outcomes}

Disease-specific survival for all Stage IV cases was $39.8 \%$ at 1 year, $19.3 \%$ at 2 years, $8.9 \%$ at 3 years, $5.3 \%$ at 4 years, and $3.3 \%$ at 5 years. The median survival time (MST) was 9 months. Over the maximal follow-up of 121 months, 245 deaths occurred; $236(96.3 \%)$ were due to gastric cancer, and $9(3.7 \%)$ were not due to gastric cancer (other disease $(n=7)$, other malignancy $(n=1)$, post-operative complication $(\mathrm{n}=1))$.

\subsection{Surgery and Clinical Characteristics}

Associations between surgery and clinical characteristics 
were compared by univariate analysis. Surgery was associated with liver metastasis, peritoneal metastasis, distant organ metastasis, and the number of Stage IV factors (Table 1). Multivariate analysis showed that surgery was significantly associated with distant organ metastasis.
The resection rate of cases with no distant organ metastasis (M0) was $89.8 \%$, but in cases with distant organ metastasis (M1), the resection rate was only $34.6 \%$. The resection rate was significantly higher for $\mathrm{M} 0$ cases than for M1 cases $(P<0.001)$ (Table 2).

Table 1. Correlations between resection and clinical factors.

\begin{tabular}{|c|c|c|c|c|}
\hline factor & No. & $(-)(n=65)$ & $(+)(n=239)$ & $P$ value \\
\hline Sex & & & & 0.754 \\
\hline male & 206 & $43(66.2 \%)$ & $163(68.2 \%)$ & \\
\hline female & 98 & $22(33.8 \%)$ & $76(31.8 \%)$ & \\
\hline Age/years old & & & & 0.844 \\
\hline$\geq 60$ & 220 & $46(71.9 \%)$ & $174(73.1 \%)$ & \\
\hline$<60$ & 82 & $18(28.1 \%)$ & $64(26.9 \%)$ & \\
\hline Macroscopic type & & & & 0.639 \\
\hline Type 0 & 4 & $1(1.7 \%)$ & $3(1.3 \%)$ & \\
\hline 1,2 & 64 & $13(22.0 \%)$ & $51(21.3 \%)$ & \\
\hline 3,4 & 215 & $46(72.9 \%)$ & $172(72.0 \%)$ & \\
\hline 5 & 15 & $2(3.4 \%)$ & $13(5.4 \%)$ & \\
\hline Site & & & & 0.664 \\
\hline L (lower) & 121 & $24(40.0 \%)$ & $97(40.6 \%)$ & \\
\hline M (middle) & 99 & $18(30.0 \%)$ & $81(33.9 \%)$ & \\
\hline U (upper) & 79 & $18(30.0 \%)$ & $61(25.5 \%)$ & \\
\hline The number of sites & & & & 0.801 \\
\hline 1 & 64 & $14(23.3 \%)$ & $50(20.9 \%)$ & \\
\hline 2 & 103 & $17(28.3 \%)$ & $86(36.0 \%)$ & \\
\hline$\geq 3$ & 132 & $29(48.4 \%)$ & $103(43.1 \%)$ & \\
\hline $\mathrm{H}$ (liver metastasis) & & & & $<0.001^{*}$ \\
\hline 0 & 216 & $27(54.0 \%)$ & $189(79.1 \%)$ & \\
\hline 1 & 73 & $23(46.0 \%)$ & $50(20.9 \%)$ & \\
\hline $\mathrm{P}$ (peritoneal metastasis) & & & & $0.008^{*}$ \\
\hline 0 & 137 & $15(30.0 \%)$ & $122(51.0 \%)$ & \\
\hline 1 & 152 & $35(70.0 \%)$ & $117(49.0 \%)$ & \\
\hline M1(LYM) (distant lymph node metastasis) & & & & 0.198 \\
\hline$(-)$ & 174 & $23(56.1 \%)$ & $151(64.3 \%)$ & \\
\hline$(+)$ & 102 & $18(43.9 \%)$ & $84(35.7 \%)$ & \\
\hline M (distant organ metastasis) & & & & $<0.001^{*}$ \\
\hline 0 & 255 & $25(59.5 \%)$ & $230(96.2 \%)$ & \\
\hline 1 & 26 & $17(40.5 \%)$ & $9(3.8 \%)$ & \\
\hline Depth & & & & 0.143 \\
\hline $\mathrm{T} 1, \mathrm{~T} 2, \mathrm{~T} 3$ & 12 & $1(2.5 \%)$ & $11(4.7 \%)$ & \\
\hline $\mathrm{T} 4 \mathrm{a}$ & 207 & $27(67.5 \%)$ & $180(75.3 \%)$ & \\
\hline $\mathrm{T} 4 \mathrm{~b}$ & 60 & $12(30.0 \%)$ & $48(20.1 \%)$ & \\
\hline Chemotherapy & 109 & & & 0.294 \\
\hline$(-)$ & 109 & $26(41.9 \%)$ & $83(34.7 \%)$ & \\
\hline$(+)$ & 192 & $36(58.1 \%)$ & $156(65.3 \%)$ & \\
\hline Stage IV factors & 241 & & & $<0.001^{*}$ \\
\hline 1 & 241 & $39(60.0 \%)$ & $202(84.5 \%)$ & \\
\hline 2 & 63 & $26(40.0 \%)$ & $37(15.5 \%)$ & \\
\hline New anti-cancer drug & & & & 0.266 \\
\hline$(-)$ & 78 & $13(36.1 \%)$ & $65(41.7 \%)$ & \\
\hline$(+)$ & 114 & $23(63.9 \%)$ & $91(58.3 \%)$ & \\
\hline
\end{tabular}

${ }^{*} \mathrm{P}<0.05$ indicates statistical significance; The incidences in some cases were unknown. 
Table 2. Multivariate analysis between resection and clinical factors.

\begin{tabular}{|c|c|c|c|c|c|c|c|c|}
\hline Variables & Resection (-) & Resection $(+)$ & B & SE & Wald & df & $P$ & $\operatorname{Exp}(B)$ \\
\hline \multicolumn{9}{|l|}{$\mathrm{H}$} \\
\hline H0 (216) & $27(13.0 \%)$ & $189(87.0 \%)$ & -0.455 & 0.552 & 0.681 & 1 & 0.409 & 0.634 \\
\hline H1 (73) & $23(31.5 \%)$ & $50(68.5 \%)$ & & & & & & \\
\hline \multicolumn{9}{|l|}{$\mathrm{P}$} \\
\hline P0 (137) & $15(10.9 \%)$ & $122(89.1 \%)$ & -0.509 & 0.494 & 1.062 & 1 & 0.303 & 0.601 \\
\hline P1 (152) & $35(23.0 \%)$ & $117(77.0 \%)$ & & & & & & \\
\hline \multicolumn{9}{|l|}{ M } \\
\hline M0 (255) & $25(10.2 \%)$ & $230(89.8 \%)$ & -2.214 & 0.619 & 12.809 & 1 & $<0.001^{*}$ & 0.109 \\
\hline M1 (26) & $17(65.4 \%)$ & $9(34.6 \%)$ & & & & & & \\
\hline \multicolumn{9}{|c|}{ Stage IV factors } \\
\hline $1(241)$ & $39(16.6 \%)$ & $202(83.4 \%)$ & -0.417 & 0.584 & 0.51 & 1 & 0.475 & 0.659 \\
\hline$\geqq 2(63)$ & $26(41.3 \%)$ & $37(58.7 \%)$ & & & & & & \\
\hline
\end{tabular}

B: coefficient for the constant; SE: standard error; Wald: Wald chi-square test; df: degree of freedom; Exp(B): exponentiation of the B coefficient; H: liver metastasis; $P$ : peritoneal metastasis; M: distant organ metastasis; ${ }^{*} P<0.05$ indicates statistical significance.

\subsection{Stage IV Gastric Cancer Disease-Specific Survival}

Using the log-rank test, macroscopic type, number of sites, peritoneal metastases, depth of invasion, resection, chemotherapy, and the number of Stage IV factors affected survival (Table 3). Multivariate analysis showed that chemotherapy [HR $0.301(95 \%$ CI $0.220-0.411 P<$ $0.001)$ ] and the number of Stage IV factors [HR 1.736 (95\%CI $1.194-2.524 P=0.004)]$ were independent prognostic factors (Table 3 ).

The SMT of chemotherapy cases was 13 months, and that of non-chemotherapy cases was 5 months. The SMT of cases with one Stage IV factor was 11 months, and that of cases with multiple Stage IV factors was 4 months.

\subsection{Stage IV Gastric Cancer Disease-Specific Survival in the Surgery Group}

The log-rank test was used to compare the results in the surgery group, macroscopic type, peritoneal metastases, depth of invasion, histology, tumor size, venous invasion, proximal margin, chemotherapy, number of Stage IV factors, lymph node dissection, and residual tumor affected the prognosis (Table 4). Multivariate analysis showed that venous invasion [HR 1.418 (95\% CI 1.009 $1.992 P=0.044)$ ], chemotherapy [HR $0.320(95 \% \mathrm{CI}$ $0.228-0.448 P<0.001)$ ], and residual tumor [HR 1.314 $(95 \%$ CI $1.055-1.638 P=0.015)]$ were each independent prognostic factors (Table 4). Examining the survival curves according to histology, the SMT of differentiated type was 14 months, and that of undifferentiated type was 8 months. Examining the survival curves of venous invasion, the SMT of $\mathrm{v} 0, \mathrm{v} 1$ cases was 11 months, and that of v2, v3 cases was 7 months. Examining the survival curves by chemotherapy in the surgery group, the
SMT of chemotherapy cases was 14 months, and that of non-chemotherapy cases was 6 months. Examining of the survival cases of residual tumor, the SMT of R0 cases was 14 months, and that of R1 cases was 15 months, and that of R2 cases was 8 months.

\subsection{Stage IV Gastric Cancer Disease-Specific Survival in the R2 Group}

The log-rank test was used in the R2 group to investigate macroscopic type, histology, tumor size, proximal margin, chemotherapy, number of Stage IV factors, and new drugs affected the prognosis (Table 5). Multivariate analysis showed that new drugs [HR 0.582 (95\% 0.363 $0.933 P=0.025)]$ were each independent prognostic factors (Table 5). Examining the survival curves according to anti-cancer drug, the SMT of the conventional anticancer drug group was 7 months, and that of the new anti-cancer drug group was 19 months.

\subsection{The Survival Curves of Surgery with Chemotherapy vs. Chemotherapy}

The SMT was 9 months in the chemotherapy alone group, 13 months in the R2 with chemotherapy group, 14 months in the R1 with chemotherapy group, and 27 months in the R0 with chemotherapy group. There was no significant difference in the survival curves between the chemotherapy alone group and the R2 with chemotherapy group $(P=0.2731)$. The survival curve of the $\mathrm{R} 1$ with chemotherapy group tended to be higher than that of the chemotherapy alone group $(P=0.0761)$. The survival curve of the R0 with chemotherapy group was significantly higher than that of the chemotherapy alone group $(P=0.0040)$. 
Table 3. The disease specific survival in all cases.

\begin{tabular}{|c|c|c|c|c|c|c|}
\hline \multirow[b]{2}{*}{ Variables } & \multicolumn{3}{|c|}{ Univariate analysis (log-rank test) } & \multicolumn{3}{|c|}{ Multivariate analysis (Cox proportional hazards model) } \\
\hline & Statistic & df & $P$ & Hazard ratio & $95 \% \mathrm{CI}$ & $P$ \\
\hline Sex & 1.35 & 1 & 0.2446 & & & \\
\hline Age & 0.39 & 1 & 0.5302 & & & \\
\hline Macro type & 11.81 & 3 & 0.0081 & 1.408 & $0.970-2.043$ & 0.072 \\
\hline Site & 0.50 & 2 & 0.7769 & & & \\
\hline The number of site & 6.04 & 2 & 0.0488 & 1.050 & $0.872-1.265$ & 0.605 \\
\hline $\mathrm{H}$ & 1.22 & 1 & 0.2699 & & & \\
\hline $\mathrm{P}$ & 16.51 & 1 & $<0.0001$ & 1.115 & $0.829-1.499$ & 0.472 \\
\hline M1(LYM) & 0.14 & 1 & 0.7084 & & & \\
\hline M1 & 2.84 & 1 & 0.0921 & & & \\
\hline Depth & 11.26 & 2 & 0.0036 & 1.330 & $0.989-1.789$ & 0.059 \\
\hline Resection & 13.52 & 1 & 0.0002 & 0.920 & $0.576-1.468$ & 0.726 \\
\hline Chemotherapy & 84.55 & 1 & $<0.0001$ & 0.301 & $0.220-0.411$ & $<0.001^{*}$ \\
\hline Stage IV factors & 17.20 & 1 & $<0.0001$ & 1.736 & $1.194-2.524$ & $0.004^{*}$ \\
\hline New drug & 1.55 & 1 & 0.2130 & & & \\
\hline
\end{tabular}

Df: degree of freedom; CI: confidential interval; H: liver metastasis; $P$ : peritoneal metastasis; M1(LYM): distant lymph node metastasis; M1: distant organ metastasis; ${ }^{*} P<0.05$ indicates statistical significance.

Table 4. The disease specific survival in surgery cases.

\begin{tabular}{|c|c|c|c|c|c|c|}
\hline \multirow[b]{2}{*}{ Variables } & \multicolumn{3}{|c|}{ Univariate analysis (log-rank test) } & \multicolumn{3}{|c|}{ Multivariate analysis (Cox proportional hazards model) } \\
\hline & Statistic & df & $P$ & Hazard ratio & $95 \% \mathrm{CI}$ & $P$ \\
\hline Sex & 2.56 & 1 & 0.1094 & & & \\
\hline Age & 1.43 & 1 & 0.2315 & & & \\
\hline Macro type & 10.02 & 3 & 0.0184 & 1.452 & $0.953-2.214$ & 0.083 \\
\hline Site & 2.11 & 2 & 0.3476 & & & \\
\hline The number of site & 4.92 & 2 & 0.0854 & & & \\
\hline $\mathrm{H}$ & 0.28 & 1 & 0.5989 & & & \\
\hline $\mathrm{P}$ & 8.41 & 1 & 0.0037 & 1.012 & $0.708-1.447$ & 0.947 \\
\hline $\mathrm{N}$ & 3.11 & 2 & 0.2115 & & & \\
\hline M1(LYM) & 0.22 & 1 & 0.6353 & & & \\
\hline M1 & 0.04 & 1 & 0.8494 & & & \\
\hline $\mathrm{CY}$ & 0.67 & 1 & 0.4142 & & & \\
\hline Depth & 8.92 & 2 & 0.0116 & 1.281 & $0.931-1.763$ & 0.128 \\
\hline Histology & 11.85 & 1 & 0.0079 & 1.294 & $0.936-1.789$ & 0.119 \\
\hline Size & 14.96 & 1 & 0.0001 & 1.233 & $0.877-1.734$ & 0.229 \\
\hline Stroma & 0.27 & 2 & 0.8759 & & & \\
\hline $\mathrm{INF}$ & 3.36 & 2 & 0.1867 & & & \\
\hline ly & 5.43 & 2 & 0.0663 & & & \\
\hline $\mathrm{v}$ & 5.35 & 1 & 0.0208 & 1.418 & $1.009-1.992$ & $0.044^{*}$ \\
\hline DM & 3.23 & 1 & 0.0722 & & & \\
\hline PM & 14.38 & 1 & 0.0001 & 1.365 & $0.812-2.294$ & 0.241 \\
\hline Chemotherapy & 65.23 & 1 & $<0.0001$ & 0.320 & $0.228-0.448$ & $<0.001^{*}$ \\
\hline Stage IV factors & 13.20 & 1 & 0.0003 & 1.497 & $0.950-2.358$ & 0.082 \\
\hline Neoadjuvant & 3.20 & 1 & 0.0739 & & & \\
\hline New drug & 1.40 & 1 & 0.2367 & & & \\
\hline LN dissection & 14.07 & 2 & 0.0028 & 1.115 & $0.924-1.346$ & 0.257 \\
\hline $\mathrm{R}$ & 18.05 & 2 & 0.0001 & 1.314 & $1.055-1.638$ & $0.015^{*}$ \\
\hline
\end{tabular}

Df: degree of freedom; CI: confidential interval; H: liver metastasis; P: peritoneal metastasis; N: extent of lymph node metastasis; M1(LYM): distant lymph node metastasis; M1: distant organ metastasis; CY: peritoneal cytology; INF: pattern of infiltrating growth; ly: lymphatic invasion; v: venous invasion; DM: distal margin; PM: proximal margin; R: residual tumor; ${ }^{*} \mathrm{P}<0.05$ indicates statistical significance. 
Table 5. The disease specific survival in $\mathrm{R} 2$ cases.

\begin{tabular}{|c|c|c|c|c|c|c|}
\hline \multirow[b]{2}{*}{ Variables } & \multicolumn{3}{|c|}{ Univariate analysis (log-rank test) } & \multicolumn{3}{|c|}{ Multivariate analysis (Cox proportional hazards model) } \\
\hline & Statistic & $\mathrm{df}$ & $P$ & Hazard ratio & $95 \% \mathrm{CI}$ & $P$ \\
\hline Sex & 2.27 & 1 & 0.1315 & & & \\
\hline Age & 1.81 & 1 & 0.1780 & & & \\
\hline Macro type & 9.19 & 3 & 0.0269 & 1.827 & $0.886-3.776$ & 0.103 \\
\hline Site & 0.76 & 2 & 0.6849 & & & \\
\hline The number of site & 1.79 & 2 & 0.4095 & & & \\
\hline $\mathrm{H}$ & 0.78 & 1 & 0.3782 & & & \\
\hline $\mathrm{P}$ & 3.66 & 1 & 0.0558 & & & \\
\hline $\mathrm{N}$ & 4.57 & 2 & 0.1017 & & & \\
\hline M1(LYM) & 0.00 & 1 & 0.9768 & & & \\
\hline M1 & 0.21 & 1 & 0.8380 & & & \\
\hline Depth & 0.81 & 2 & 0.6672 & & & \\
\hline Histology & 7.04 & 1 & 0.0295 & 1.328 & $0.828-2.131$ & 0.239 \\
\hline Size & 3.88 & 1 & 0.0488 & 0.937 & $0.570-1.538$ & 0.796 \\
\hline Stroma & 0.31 & 2 & 0.8561 & & & \\
\hline INF & 0.27 & 2 & 0.8726 & & & \\
\hline ly & 4.87 & 2 & 0.0876 & & & \\
\hline $\mathrm{v}$ & 0.69 & 1 & 0.4065 & & & \\
\hline DM & 0.45 & 1 & 0.5028 & & & \\
\hline PM & 7.32 & 1 & 0.0068 & 2.013 & $0.817-4.958$ & 0.128 \\
\hline Chemotherapy & 38.00 & 1 & $<0.0001$ & - & - & - \\
\hline Stage IV factors & 4.23 & 1 & 0.0396 & 1.349 & $0.737-2.470$ & 0.332 \\
\hline neoadjuvant & 1.82 & 1 & 0.1778 & & & \\
\hline New drug & 4.48 & 1 & 0.0343 & 0.582 & $0.363-0.933$ & $0.025^{*}$ \\
\hline LN dissection & 2.93 & 2 & 0.4018 & & & \\
\hline
\end{tabular}

Df: degree of freedom; CI: confidential interval; H: liver metastasis; $P$ : peritoneal metastasis; N: extent of lymph node metastasis; M1(LYM): distant lymph node metastasis; M1: distant organ metastasis; CY: peritoneal cytology; INF: pattern of infiltrating growth; ly: lymphatic invasion; v: venous invasion; DM: distal margin; PM: proximal margin; ${ }^{*} \mathrm{P}<0.05$ indicates statistical significance.

\subsection{Residual Tumor by Treatment}

Examining the rate of residual tumor according to treatment, in the surgery alone group, R0 was $13.4 \%(\mathrm{n}=11)$, R1 was $4.9 \%(n=4)$, and R2 was $81.7 \%(n=67)$. In the postoperative chemotherapy group, R0 was $21.6 \%(\mathrm{n}=$ 29), $R 1$ was $14.9 \%(n=20)$, and $R 2$ was $63.4 \%(n=12)$. In the preoperative chemotherapy group, R0 was $36.4 \%$ $(\mathrm{n}=8), \mathrm{R} 1$ was $9.1 \%(\mathrm{n}=2)$, and $\mathrm{R} 2$ was $54.5 \%(\mathrm{n}=12)$. The R0 rate was significantly higher in the preoperative chemotherapy group than in the surgery alone group $(P=$ 0.048) (Table 6).

\subsection{The Regimens of the Preoperative Chemotherapy Group}

The mean numbers of administered courses of 5-FU/ CDDP, S-1/CDDP, S-1/TXL, and S-1 were 1.6, 3.4, 5.5, and 2.5 , respectively. The rates of down staging were
$22.2 \%(\mathrm{n}=2), 25.0 \%(\mathrm{n}=2), 50.0 \%(\mathrm{n}=1)$, and 0 , respectively. The rates of R0 or R1 were $55.6 \%(n=5)$, $62.5 \%(\mathrm{n}=5), 50.0 \%(\mathrm{n}=1)$, and 0 , respectively. The response rates were $77.8 \%(n=7), 50.0 \%(n=4), 100 \%$ $(\mathrm{n}=2)$, and $50.0 \%(\mathrm{n}=1)$, respectively. The rates of patients who underwent postoperative chemotherapy using the $\mathrm{S}-1$ regimen were $0,87.5 \%(\mathrm{n}=7), 100 \%(\mathrm{n}=$ $2)$, and $100 \%(\mathrm{n}=2)$, respectively. No cases underwent postoperative chemotherapy using the S-1 regimen in the 5-FU/CDDP group, because the cases given 5-FU/CDDP were old cases. There were four 2-year survival cases $(50.0 \%)$ with S-1/CDDP, but there was no 2-year survival case with the other regimens (Table 7).

\subsection{The S-1/CDDP Regimen in the Preoperative Chemotherapy Group}

With the S-1/CDDP regimen, there was no case with distant organ metastases. There were three PR cases, and 
Table 6. Residual tumor after surgery.

\begin{tabular}{ccccc}
\hline & R0 & R1 & R2 & Total \\
\hline Surgery alone & $11^{*}(13.4 \%)$ & $4(4.9 \%)$ & $67(81.7 \%)$ & $82(100 \%)$ \\
Postoperative-chemotherapy & $29(21.6 \%)$ & $20(14.9 \%)$ & $85(63.4 \%)$ & $134(100 \%)$ \\
Preoperative-chemotherapy & $8^{*}(36.4 \%)$ & $2(9.1 \%)$ & $12(54.5 \%)$ & $22(100 \%)$ \\
Total & 48 & 26 & 164 & 238 \\
\hline
\end{tabular}

R0: no residual tumor; R1: microscopic residual tumor (positive resection margin or CY1); R2: macroscopic residual tumor; ${ }^{*}$ : The rate of preoperative chemotherapy cases is significantly higher than that of surgery alone cases $(P=0.048)$.

Table 7. The regimen of preoperative chemotherapy.

\begin{tabular}{ccccccc}
\hline & Cycle & Down stage & R0,R1 & Response & S-1(post ope.) & 2 yr. survival \\
\hline 5-FU/CDDP $(\mathrm{n}=9)$ & 1.6 & $2(22.2 \%)$ & $5(55.6 \%)$ & $7(77.8 \%)$ & 0 & 0 \\
S-1/CDDP $(\mathrm{n}=8)$ & 3.4 & $2(25.0 \%)$ & $5(62.5 \%)$ & $4(50.0 \%)$ & $7(87.5 \%)$ & $4(50.0 \%)$ \\
S-1/TXL $(\mathrm{n}=2)$ & 5.5 & $1(50.0 \%)$ & $1(50.0 \%)$ & $2(100 \%)$ & $2(100 \%)$ & 0 \\
S-1 $(\mathrm{n}=2)$ & 2.5 & 0 & 0 & $1(50.0 \%)$ & $2(50.0 \%)$ & 0 \\
\hline
\end{tabular}

R0: no residual tumor; R1: microscopic residual tumor (positive resection margin or CY1); 5-FU: 5-fluorouracil; CDDP: cisplatin; TXL: taxol.

$1 \mathrm{SD}$ case in the 2-year survival cases. Three of four PR cases were 2-year survival cases, and another PR case with $\mathrm{R} 0$ resection died from other disease at 9 months after surgery. One R0, two R1, and one R2 case were 2-year survival cases, but the R2 case died of liver metastases at 25 months after surgery. The histological responses of the two 2-year survival cases were grade 2 . All four 2-year survival cases underwent postoperative chemotherapy using S-1 with CDDP or TXL (Table 8).

\section{Discussion}

In this paper, the effect of surgery on the prognosis of Stage IV gastric cancer was examined. According to the guidelines for the treatment of gastric cancer (3rd Edition), the treatments for Stage IV gastric cancer are chemotherapy, radiation, palliative surgery, and symptommatic treatment [9]. The chief treatment for Stage IV gastric cancer is chemotherapy, but palliative surgery for stenosis or bleeding due to the tumor is recommended. The survival benefit of reduction surgery is controversial. Chemotherapy plays an important role in the treatment of Stage IV gastric cancer, but no effective chemotherapy regimen for unresectable or recurrent gastric carcinoma was established until the 1990s. S-1, a new oral fluoropyrimidine containing tegafur, 5-chloro-2, 4-dihydropyrimidine, and potassium oxonate, was recognized as an effective drug for gastric cancer in the mid 1990s [10]. Recently, the Japanese ACTS-GC trial demonstrated the efficacy of S-1 for Stage II-III gastric cancer patients after a curative resection with a D2 lymphadenectomy, where S-1 was found to improve the 3-year overall survival (OS) from $70.1 \%$ for surgery alone to $80.1 \%$ [11]. Several novel chemotherapeutic agents, including irinotecan (CPT-11), taxanes (paclitaxel and docetaxel), and oxaliplatin in combination with S-1, have demonstrated beneficial activity against gastric cancer and offer hope for improving patient outcomes [12-14]. Several chemotherapy regimens, such as S-1 plus cisplatin of the SPIRITS trial, have recently shown a remarkably high response rate [15], and this regimen is frequently used as first-line chemotherapy, with good results in advanced cases [16]. In addition, some molecular-targeting agents have been investigated for gastric cancer [11,13], and these agents are expected to make revolutionary progress in chemotherapy for unresectable or recurrent gastric cancer [17]. The ToGA trial was the first randomized trial investigating anti-HER2 therapy in advanced gastric cancer; it showed that trastuzumab (herceptin) with chemotherapy (5-fluorouracil (5-FU) or capecitabine and cisplatin) was superior to chemotherapy alone [18]. These new anti-cancer drugs and molecular-targeting agents are thought to be contributing to significant improvements in anti-tumor responses and patient survival.

In the present study, chemotherapy and the number of Stage IV factors were independent prognostic factors in all Stage IV cases. Surgery was significant on univariate analysis, but it was not an independent prognostic factor. These results suggest that the chief therapy for Stage IV gastric cancer was thought to be chemotherapy. In surgery cases, venous invasion, chemotherapy, and residual tumor were independent prognostic factors. Therefore, chemotherapy with resection might be an effective therapy for Stage IV gastric cancer. There was no significant difference in prognosis between chemotherapy with R1 or R2 resection cases and chemotherapy alone cases. However, the prognosis of chemotherapy with R0 resection cases was significantly better than that of chemotherapy alone cases. Chemotherapy with R0 resection was considered to improve the prognosis of Stage IV 
Table 8. Patients who underwent preoperative chemotherapy using S-1/CDDP.

\begin{tabular}{|c|c|c|c|c|c|c|c|c|}
\hline No. & cycle & type & cStage & ypStage & $\mathrm{R}$ & efficacy & chem. after surgery & prognosis \\
\hline 1 & 5 & 3 & T4bH1P0M1LYM IV & T1bN0H1P0CY0 IV & 2 & PR & $\mathrm{S}-1 / \mathrm{CDDP}$ & $25 \mathrm{M}$ dead \\
\hline 2 & 4 & 4 & $\mathrm{~T} 4 \mathrm{bN}(+) \mathrm{H} 0 \mathrm{P} 1 \mathrm{IV}$ & T4aN1H0P0CY1 IV & 1 & PR & $\mathrm{S}-1 / \mathrm{CDDP}$ & $29 \mathrm{M}$ alive \\
\hline 3 & 6 & 2 & T4aH0P0M1LYM IV & T2N2H0P0CY0 IIB & 0 & PR & $\mathrm{S}-1 / \mathrm{CDDP}$ & $32 \mathrm{M}$ alive \\
\hline 4 & 2 & 3 & T4aH0P0M1LYM IV & T2N3H0P0CY0 IIIA & 0 & PR & S-1 & $9 \mathrm{M}$ other \\
\hline 5 & 2 & 4 & T4aN1H0P0M0 IIIA & T4aN0H0P0CY1 IV & 1 & SD & S-1/CDDP & $8 \mathrm{M}$ dead \\
\hline 6 & 4 & 3 & T4aN3H0P0M0 IIIC & T4aN3H1P0CY0 IV & 2 & $\mathrm{PD}$ & S-1 & $6 \mathrm{M}$ dead \\
\hline 7 & 2 & 4 & T4aN3H0P0M0 IIIC & T4aN3H0P0CY1 IV & 1 & SD & S-1/TXL & $29 \mathrm{M}$ alive \\
\hline 8 & 2 & 4 & T4aH0P0M1LYM IV & T4aN3H0P1CY1 IV & 2 & PD & - & $2 \mathrm{M}$ dead \\
\hline
\end{tabular}

cStage: clinical stage before chemotherapy; ypStage: pathological stage after surgery; R: residual tumor; chem. R0: no residual tumor; R1: microscopic residual tumor (positive resection margin or CY1); R2: macroscopic residual tumor; PR: partial response; SD: stable disease, PD: progressive disease; chem.: chemotherapy; CDDP: cisplatin; TXL: taxol; M: months after surgery; other: death due to other disease.

gastric cancer. Smith et al. [19] demonstrated that, among all patients with Stage IV gastric cancer recommended for surgery, patients who undergo gastric resection have improved survival over those who do not undergo resection in Stage IV (AJCC staging) gastric cancer cases. However, many resection cases in Stage IV gastric cancer were R2 cases. The efficacy of R2 resection on the prognosis of Stage IV gastric cancer has been controversial. In the present study, use of new anti-cancer drugs was an independent prognostic factor in R2 cases. Therefore, further development of anti-cancer drugs and new molecular-targeting drugs is expected to increase the importance of reduction surgery for improving the prognosis of Stage IV gastric cancer in future. Okuyama et al. [20] reported that the administration of new drugs and regimens was an independent factor for the extending survival times for patients undergoing noncurative surgery.

Advances in gastric cancer chemotherapy, such as the introduction of new anticancer agents, have made macroscopic complete resection possible for some patients presenting with otherwise unresectable or metastatic gastric cancer for whom surgical resection had not been indicated at the first clinical visit. Surgical resection of a residual tumor in such patients, or the so-called salvage gastrectomy or secondary gastrectomy, has been occasionally reported since 2001. All of these reports have described surgical success and satisfactory outcomes [21-23]. The R0 rate of the preoperative chemotherapy group was significantly higher than that of the surgery alone group. Preoperative chemotherapy has several advantages over postoperative chemotherapy. First, if the case has no metastatic site target for chemotherapy on computed tomography (CT), response evaluation is possible because the primary site can be evaluated. Second, the compliance of chemotherapy is good, because there are no postoperative complications or side effects. Third, preoperative chemotherapy has a good effect on the me- tastatic site, such as bulky lymph nodes, so that the rate of curative resection is high. Fourth, anti-cancer drugs can be administered for micro-metastases in undissectable distant lymph nodes from the early phase. Fifth, useless surgery can be avoided in patients who have early recurrences after curative resection [24]. High response rates were seen in preoperative chemotherapy cases. However, there was no long survival case after surgery in the 5-FU and cisplatin regimen group. On the other hand, the 2-year survival rate was $50 \%$ in the S-1 and cisplatin group. Because all cases in the 5-FU and cisplatin regimen group were treated in the 1990s, their postoperative chemotherapies were old anti-cancer drugs, but new anti-cancer drugs, such as S-1 regimens, were administered as postoperative chemotherapy in the S-1 and cisplatin regimen group. Postoperative chemotherapy was considered to be important in preoperative chemotherapy cases, even if R0 resections were done. On the basis of the SPIRITS trial, S-1 plus cisplatin is now considered one of the preferred standard treatments for advanced gastric cancer in Japan. Treatment using new anti-cancer drugs such as S-1 and cisplatin for incurable gastric cancer patients is considered to reduce metastatic sites and/ or primary sites and increase the number of R0 resection cases, which improves the prognosis of Stage IV gastric cancer patients. Kanda et al. reported that second gastrectomy following S-1-based chemotherapy was a safe and effective treatment for Stage IV gastric cancer. DCS treatment (docetaxel, cisplatin, and S-1) was recently developed, and a clinical trial demonstrated a high response rate [25]. Oyama et al. [26] reported that preoperative DCS therapy for gastric cancer with para-aortic lymph node metastases showed high clinical response $(68.8 \%)$ and disease control rates $(100 \%)$; the pathological response rate of the resected specimens was $87.5 \%$. At 2 years after surgery, the overall survival rate was $93.8 \%$, and the relapse-free survival was $75.0 \%$. Multimodal therapy comprising combined pre-operative S-1- 
based therapy and gastrectomy was extremely effective for advanced gastric cancer and was thought to improve the prognosis of Stage IV gastric cancer patients. The importance of gastrectomy in Stage IV gastric cancer will increase as chemotherapy develops further.

However, the resection rate was very low in cases with distant organ metastases such as bone and bone marrow. Moreover, the prognosis of cases with multiple Stage IV factors was poor, and their resection rate was low. Novel strategies for treatment will be needed for cases with distant organ metastases or cases with multiple Stage IV factors.

\section{REFERENCES}

[1] D. M. Parkin, F. Bray, J. Ferlay and P. Pisani, "Global Cancer Statistics, 2002," A Cancer Journal for Clinicians, Vol. 55, No. 2, 2005, pp. 74-108. doi:10.3322/canjclin.55.2.74

[2] S. Lim, B. E. Muhs, S. G. Marcus, E. Newman, R. S. Berman and S. P. Hiotis, "Results Following Resection for Stage IV Gastric Cancer; Are Better Outcomes Observed in Selected Patient Subgroups?" Journal of Surgical Oncology, Vol. 95, No. 2, 2007, pp. 118-122. doi:10.1002/jso.20328

[3] S. Maekawa, M. Saku, Y. Maehara, N. Sadanaga, K. Ikejiri, H. Anai, H. Kuwano and K. Sugimachi, "Surgical Treatment for Advanced Gastric Cancer," Hepatogastroenterology, Vol. 43, No. 7, 1996, pp. 178-186.

[4] F. Bozzetti, G. Bonfanti, R. A. Audisio, R. Doci, G. Dossena, L. Gennari and S. Andreola, "Prognosis of Patients after Palliative Surgical Procedures for Carcinoma of the Stomach," The Journal of Surgery, Gynecology and Obstetrics, Vol. 164, No. 2, 1987, pp. 151-154.

[5] H. H. Hartgrink, H. Putter, E. Klein Kranenbarg, J. J. Bonenkamp and C. J. van de Velde, "Value of Palliative Resection in Gastric Cancer," British Journal of Surgery, Vol. 89, No. 11, 2002, pp. 1438-1443.

doi:10.1046/j.1365-2168.2002.02220.x

[6] J. J. Bonenkamp, M. Sasako, J. Hermans and C. J. van de Velde, "Tumor Load and Surgical Palliation in Gastric Cancer," Hepatogastroenterology, Vol. 48, No. 41, 2001, pp. 1219-1221.

[7] Japanese Gastric Cancer Association, "Japanese Classification of Gastric Carcinoma, 2nd English Edition," Gastric Cancer, Vol. 1, No. 1, 1998, pp. 10-24.

[8] Japanese Gastric Cancer Association, "Japanese Classification of Gastric Carvinoma, 3rd English Edition," Gastric Cancer, Vol. 14, No. 2, 2011, pp. 101-112. doi:10.1007/s10120-011-0041-5

[9] Japanese Gastric Cancer Association, "Japanese Gastric Cancer Treatment Guideline 2010 (ver. 3)," Gastric Cancer, Vol. 14, No. 2, 2011, pp. 113-123. doi:10.1007/s10120-011-0042-4

[10] Y. Maehara, "S-1 in Gastric Cancer: A Comprehensive Review," Gastric Cancer, Vol. 6, Suppl. 1, 2003, pp. 2-8. doi:10.1007/s10120-003-0232-9
[11] S. Sakuramoto, M. Sasako, T. Yamaguchi, T. Kinoshita, M. Fujii, A. Nashimoto, H. Furukawa, T. Nakajima, Y. Ohashi, H. Imamura, M. Higashino, Y. Yamamura, A. Kurita and K. Arai, "Adjuvant Chemotherapy for Gastric Cancer with S-1, an Oral Fluoropyrimidine," The New England Journal of Medicine, Vol. 357, No. 18, 2007, pp. 1810-1820. doi:10.1056/NEJMoa072252

[12] Y. Emi, M. Yamamoto, I. Takahashi, H. Orita, Y. Kakeji, S. Kohnoe and Y. Maehara, "Phase II Study of Weekly Paclitaxel by One-Hour Infusion for Advanced Gastric Cancer," Surgery Today, Vol. 38, No. 11, 2008, pp. 10131020. doi:10.1007/s00595-008-3769-8

[13] M. Nishiyama and S. Wada, "Docetaxel: Its Role in Current and Future Treatments for Advanced Gastric Cancer," Gastric Cancer, Vol. 12, No. 3, 2009, pp. 132-141. doi:10.1007/s10120-009-0521-Z

[14] E. Van Cutsem, D. Haller and A. Ohtsu, "The Role of Chemotherapy in Current Treatment of Gastric Cancer," Gastric Cancer, Vol. 5, Suppl. 1, 2002, pp. 17-22. doi:10.1007/s10120-002-0219-y

[15] W. Koizumi, H. Narahara, T. Hara, A. Takagane, T. Akiya, M. Takagi, K. Miyashita, T. Nishizaki, O. Kobayashi, W. Takiyama, Y. Toh, T. Nagaie, S. Takagi, Y. Yamamura, K. Yanaoka, H. Orita and M. Takeuchi, "S-1 plus Cisplatin versus S-1 Alone for First-Line Treatment of Advanced Gastric Cancer (SPIRITS trial): A Phase III Trial," The Lancet Oncology, Vol. 9, No. 3, 2008, pp. 215-221. doi:10.1016/S1470-2045(08)70035-4

[16] A. Nashimoto, H. Yabusaki, S. Nakagawa, Y. Takii, Y. Tsuchiya and T. Otsuo, "Preoperative Chemotherapy with S-1 and Cisplatin for Highly Advanced Gastric Cancer," Anticancer Research, Vol. 29, No. 11, 2009, pp. 46894696.

[17] M. Fujii, M. Kochi and T. Takayama, "Recent Advances in Chemotherapy for Advanced Gastric Cancer in Japan," Surgery Today, Vol. 40, No. 4, 2010, pp. 295-300. doi:10.1007/s00595-009-4148-9

[18] Y. J. Bang, E. Van Cutsem, A. Fevereislova, H. C. Chung, L. Shen, A. Sawaki, F. Lordick, A. Ohtsu, Y. Omuro, T. Satoh, G. Aprile, E. Kulikov, J. Hill, M. Lehle, J. Rüschoff and Y. K. Kang, "Trastuzumab in Combination with Chemotherapy versus Chemotherapy Alone for Treatment of HER2-Positive Advanced Gastric or Gastro-Oesophageal Junction Cancer (ToGA): A Phase 3, Open-Label, Randomized Controlled Trial," Lancet, Vol. 376, No. 9742, 2010, pp. 687-697. doi:10.1016/S0140-6736(10)61121-X

[19] J. K. Smith, J. S. Hill, S. C. Ng, T. P. McDade, S. A. Shah and J. F. Tseng, "Potential Benefit of Resection for Stage IV Gastric Cancer: A National Survey," Journal of Gastrointestinal Surgery, Vol. 14, No. 11, 2010, pp. 1660-1668. doi:10.1007/s11605-010-1351-3

[20] T. Okuyama, D. Korenaga, K. Koushi, S. Itoh, H. Kawanaka, Y. Ikeda and K. Takenaka, "The Prognostic Significance of Chemotherapy for Stage IV Gastric Cancer Patients: A Single-Institution Experience," Surgery Today, Vol. 41, No. 7, 2011, pp. 935-940. doi:10.1007/s00595-010-4403-0

[21] M. Iwahashi, M. Nakamori, M. Tani, H. Yamaue, S. 
Sakaguchi, M. Nakamura, K. Ueda, M. Ichiro, E. Nishio and H. Tanimura, "Complete Response of Highly Advanced Gastric Cancer with Peritoneal Dissemination after New Combined Chemotherapy of S-1 and Low-Dose Cisplatin: Report of a Case," Oncology, Vol. 61, No. 1, 2001, pp. 16-22. doi:10.1159/000055347

[22] N. Yoshimizu, Y. Saikawa, T. Kubota, Y. Akiba, M. Yoshida, Y. Otani, K. Kumai, T. Hibi and M. Kitajima, "Complete Response of a Highly Advanced Gastric Carcinoma to Preoperative Chemoradiotherapy with S-1 and Low Dose Cisplatin," Gastric Cancer, Vol. 6, No. 3, 2003, pp. 185-190. doi:10.1007/s10120-003-0239-2

[23] I. Yoshida, Y. Sakurai, Y. Komori, S. Tonomura, T. Masui, M. Shoji, Y. Nakamura, H. Imazu, I. Uyama and M. Ochiai, "Successful Downstaging by S-1 Based Chemotherapy Followed by Surgical Resections for Gastric Carcinoma with Extensive Distant Lymph Node Metastasis-Report of Two Cases and a Review of Cases with Surgical Resection after Downstaging by S-1 Based Chemotherapy," Hepatogastroenterology Vol. 52, No. 63,

\section{Abbreviation}

$\mathrm{L}$ : lower third portion

$\mathrm{M}$ : middle third portion

$\mathrm{U}$ : upper third portion

$\mathrm{H}$ : hepatic metastasis

P: peritoneal metastasis

$\mathrm{N}$ : lymph node metastasis

M: distant metastasis

LYM: lymph node

CY: peritoneal lavage cytology

$\mathrm{T}$ : depth of tumor invasion

medullary type (med): scanty stroma

scirrhous type (sci): abundant stroma

intermediate type (int): the quantity stroma is

intermediate between the two above type

INFa: tumor displays expanding growth with a distant
2005, pp. 978-984.

[24] A. Tsuburaya, "Adjuvant Chemotherapy for Gastric Cancer: Current Status and Future Perspective," Gastroenterological Surgery, Vol. 35, No. 2, 2012, pp. 193-200.

[25] T. Kanda, K. Yajima, S. Kosugi, T. Ishikawa, Y. Ajioka and K. Hatakeyama, "Gastrectomy as a Secondary Surgery for Stage IV Gastric Cancer Patients Who Underwent S-1-Based Chemotherapy: A Multi-Institute Retrospective Study," Gastric Cancer, Vol. 15, No. 3, 2012, pp. 235-244. doi:10.1007/s10120-011-0100-y

[26] K. Oyama, S. Fushida, J. Kinoshita, I. Makino, K. Nakamura, H. Hayashi, H. Nakagawara, H. Tajima, H. Fujita, H. Takamura, I. Ninomiya, H. Kitagawa, T. Tani, T. Fujimura and T. Ohta, "Efficacy of Pre-Operative Chemotherapy with Docetaxel, Cisplatin, and S-1 (DCS Therapy) and Curative Resection for Gastric Cancer with Pathologically Positive Para-Aortic Lymph Nodes," Journal of Surgical Oncology, Vol. 105, No. 6, 2012, pp. 535-541. doi:10.1002/jso.22125

border from the surroundinr tissue

$\mathrm{INFb}$ : tumor shows an intermediate pattern between INFa and INFc

INFc: tumor displays infiltrative growth with no distinct border with the surrounding tissue

ly: lymphatic invasion

$\mathrm{v}$ : venous invasion

PM: proximal margin

DM: distal margin

D: lymph node dissection

R0: No residual tumor

$\mathrm{R} 1$ : microscopic residual tumor

R2: macroscopic residual tumor

FU: fluorouracil

CDDP: cisplatin

TXL: taxol 